\title{
Pratiques
}

Linguistique, littérature, didactique

\section{Écrire pour enseigner : modalités du réinvestissement textuel dans les notes de cours de trois linguistes}

Writing for teaching: forms of text reuse in three linguists' lecture notes

Muriel Jorge et Pierre-Yves Testenoire

\section{(2) OpenEdition}

Journals

Édition électronique

URL : http://journals.openedition.org/pratiques/3296

DOI : $10.4000 /$ pratiques.3296

ISSN : 2425-2042

Éditeur

Centre de recherche sur les médiations (CREM)

Référence électronique

Muriel Jorge et Pierre-Yves Testenoire, «Écrire pour enseigner : modalités du réinvestissement textuel dans les notes de cours de trois linguistes », Pratiques [En ligne], 173-174 | 2017, mis en ligne le 10 mars 2017, consulté le 30 avril 2019. URL : http://journals.openedition.org/pratiques/3296 ; DOI : $10.4000 /$ pratiques.3296

Ce document a été généré automatiquement le 30 avril 2019.

(c) Tous droits réservés 


\title{
Écrire pour enseigner : modalités du réinvestissement textuel dans les notes de cours de trois linguistes
}

Writing for teaching: forms of text reuse in three linguists' lecture notes

\author{
Muriel Jorge et Pierre-Yves Testenoire
}

Les processus à l'œuvre dans la production de textes scientifiques ont fait l'objet, ces dernières années, d'un nombre important de travaux de la part d'historiens et de sociologues des sciences (Jacob, 2011; Bert, 2014 ; Waquet, 2015), de généticiens du texte (Barberousse \& Pinon, 2003 ; Fenoglio, 2012) et de linguistes (Mondada, 2005 ; DoquetLacoste, 2009; Chepiga \& Sofia, 2014). La découverte d'un nombre croissant de fonds d'archives de chercheurs ouvre sur ces questions de nouveaux observatoires. Leur prise en compte permet d'établir dans la diversité des gestes qui font le quotidien de l'activité savante - écriture d'un article, préparation d'un cours ou d'une conférence, travail de terrain... - des invariants et des spécificités propres à chaque scripteur.

Une constante des fonds d'archives scientifiques modernes est la réutilisation incessante des matériaux écrits. Plus que la simple réutilisation du papier (découpage de manuscrits pour en faire des petites fiches ou écriture au verso d'un jeu d'épreuves corrigées par exemple), c'est le réinvestissement d'écrits antérieurs dans un processus de textualisation qui nous préoccupera ici. Ces réemplois fréquents s'accompagnent parfois de transferts en termes de genre : des notes de cours peuvent servir à la rédaction d'une publication ou d'une conférence dans un congrès scientifique, et vice-versa ${ }^{1}$.

Dans les archives privées de chercheurs, le geste commun le plus fréquemment identifiable est la réutilisation de textes produits pour l'enseignement en vue de publier un article ou un ouvrage de référence. Pour prendre quelques exemples parmi les linguistes, on pensera à $\mathrm{F}$. Brunot, qui publie « le cours de langue qu['il] faisai[t] à l'École de Sèvres" sous le titre La pensée et la langue (1922). De même, A. Meillet dit avoir rassemblé ses idées «à l'occasion d'un cours fait au Collège de France en 1921-1922 » 
avant d'écrire Les origines indo-européennes des mètres grecs (1923). On retrouve également cet usage chez C. Bally, J. Vendryes, É. Benveniste ${ }^{2}$...

On s'intéressera ici au phénomène inverse, à savoir le réinvestissement textuel d'écrits antérieurs dans l'écriture à visée didactique. Se pencher sur la "littérature grise » que constituent les archives d'enseignants est l'occasion de dépasser l'aspect immédiatement perceptible du cours en tant que mode de diffusion et de transmission d'un savoir "disponible", pour s'interroger sur la construction de ce qui est «mis à disposition" (Puech \& Chiss, 1998). On observera pour cela les manuscrits de trois linguistes de langue française qui réutilisent des écrits antérieurs dans la préparation de leurs cours: A. Darmesteter (1846-1888), F. Brunot (1860-1938) et C. Bally (1865-1947). Ce corpus se caractérise par une triple homogénéité: chronologique (il couvre un empan chronologique qui va des années 1870 aux années 1930, qui correspond, dans l'enseignement supérieur en France et à Genève, à un renforcement du lien entre enseignement et recherche), documentaire (il s'agit de manuscrits de travail, conservés à Genève et à Paris ${ }^{3}$, et spécifiquement d'écrits à visée didactique) et disciplinaire (il porte sur la linguistique). Notre objectif n'est pas de dégager quelques traits idiosyncrasiques dans la pratique de tel ou tel savant mais plutôt de repérer les modalités récurrentes de la réutilisation de leurs propres écrits chez ces scripteurs experts et d'identifier le rôle de la perspective didactique dans ce processus.

\section{Le réinvestissement textuel : problèmes théoriques et méthodologiques}

5 Parler de « réinvestissement textuel » implique d'envisager deux écrits différents, qui présentent cependant des points communs. L'un des premiers problèmes à résoudre est celui de l'établissement des critères de distinction et de ressemblance entre ces écrits. On considérera ici que le réinvestissement textuel implique l'appartenance de chacun des écrits à une " phase d'écriture » différente. Afin d'identifier ces " phases ", on prendra en compte un faisceau de critères convergents, sans toutefois nier la part d'arbitraire inhérente à ce procédé. Ainsi, on entendra par «réinvestissement textuel » dans l'écriture d'un texte $B$, la mobilisation par un scripteur d'un écrit $A$, qu'il a lui-même produit lors d'une phase d'écriture antérieure, l'écrit A mobilisé - qui constitue donc un « déjà-là » du processus de textualisation de l'écrit B - pouvant être indifféremment un texte publié ou un texte manuscrit.

6 L'étude du réinvestissement textuel ainsi défini pose quantité de problèmes méthodologiques. Déterminer que deux écrits ont été produits à des moments différents ne va, en effet, pas toujours de soi. L'appartenance à deux ensembles de feuillets pochettes ou cartons - séparés suffit-elle pour juger qu'ils sont distincts l'un de l'autre? En l'absence de signes explicites, il est parfois difficile, voire impossible, de dater un feuillet, que ce soit de manière absolue ou relative. L'embarras est plus grand encore face à des morceaux découpés, collés sur un autre feuillet. La coexistence de plans, de notes éparses, de textes entièrement rédigés n'est pas toujours aisée à interpréter : est-on face au même écrit, pris à des phases de structuration différentes, ou face à deux écrits différents, l'un constituant le substrat de l'autre? Sur quelles bases affirme-t-on qu'un scripteur a réutilisé un de ses écrits pour en produire un autre? 
7 La distance temporelle qui sépare l'observateur d'aujourd'hui du moment de la production des notes de cours - dans le cas présent, entre un siècle et demi et un demisiècle - doit également être prise en compte. Entre ces deux moments s'intercalent de nombreuses étapes éventuelles : réorganisation des papiers après leur écriture par le savant lui-même, destruction de certains documents, classement ou tri effectué par les ayant-droit, dépôt éventuellement lacunaire et en plusieurs endroits, classement par un archiviste... L'identification de phénomènes de réinvestissement textuel chez tel ou tel chercheur est donc contrainte par les conditions de la conservation et de la transmission de ses archives. Par conséquent, on se gardera d'imputer, de manière absolue, à tel ou tel savant le recours ou l'absence de recours au réinvestissement textuel pour la préparation d'un cours. C'est seulement le nombre d'indices de ce phénomène laissé dans les archives qu'il est possible d'établir pour chacun.

8 Entre les enseignants-chercheurs dont les manuscrits de travail sont aujourd'hui connus, des différences notables se font jour. Les fonds d'archives font apparaitre que le réinvestissement textuel est, chez certains savants, un processus fondamental pour la préparation d'un cours, quand d'autres ne gardent de cette activité que très peu de traces. Indépendamment des conditions de conservation, plusieurs paramètres peuvent expliquer les proportions variables de réinvestissement textuel :

- Une première variable est liée aux habitudes de travail des chercheurs. Ces habitudes sont toujours singulières, même si la description d'un nombre croissant de fonds permet d'établir entre les pratiques savantes des constantes et des points de comparaison. Comme le souligne J.-F. Bert (2014 : paragr. 33-36), une part importante de l'activité d'un chercheur en sciences humaines consiste dans l'organisation de ses propres instruments de travail, ses papiers, qu'il classe ou non, selon les cas, dans des dossiers, des chemises, des enveloppes... Le chercheur apparait bien comme le premier archiviste de ses papiers, plus ou moins méticuleux, plus ou moins désinvolte. Les variations observables entre les fonds d'archives pour la préparation d'un cours concernent aussi bien les formats de papier utilisés, le protocole de travail suivi que le produit final visé (texte entièrement rédigé, plan, notes...) ${ }^{4}$. Tous ces éléments affectent les degrés de réinvestissement dans le processus d'écriture d'un cours. Ainsi l'habitude qu'ont certains enseignants-chercheurs de détruire leurs fiches de cours au fur et à mesure de leur utilisation rend difficile l'étude de leurs méthodes de préparation de leurs enseignements. C'est le cas par exemple de F.de Saussure ${ }^{5}$. Inversement les scrupules avec lesquels son disciple C. Bally, et sa veuve après lui, classent, datent et annotent l'utilisation de chaque papier pour son enseignement font de lui un très bon candidat pour l'analyse du réinvestissement textuel.

- Le deuxième paramètre majeur qui explique la propension variable des savants à réutiliser leurs écrits dépend du cadre institutionnel et social. La stabilité du public et la nature de l'établissement dans lequel l'enseignement projeté s'inscrit jouent, à cet égard, un rôle déterminant. Dans les dernières décennies du XIX ${ }^{\mathrm{e}}$ siècle, des réformes de l'enseignement supérieur en France et en Suisse modifient l'auditoire des facultés : le public cultivé des «auditeurs libres » est marginalisé au profit des étudiants régulièrement inscrits ${ }^{6}$. La mise en place dans les universités d'un public «captif ", contraint à l'assiduité par des systèmes de bourses et inscrit dans des cursus validés par des diplômes (licence de lettres, agrégation) implique la répétition, d'une année sur l'autre, des mêmes cours pour chaque nouvelle cohorte et influe nécessairement sur les degrés de renouvellement et de réemploi dans la préparation des enseignements. 
- À côté de ces paramètres externes ou sociaux, il en est un troisième, interne au champ du savoir. Ce sont, en effet, des critères d'ordre scientifique qui motivent un professeur à utiliser ou non pour un cours un de ses travaux antérieurs, à en délaisser telle ou telle partie. Les changements de positions théoriques, la prise en compte de nouvelles parutions ou de nouvelles découvertes peuvent expliquer les modifications apportées à des écrits antérieurs. Les modalités du réinvestissement textuel renseignent ainsi sur l'évolution des parcours de recherche et d'enseignement. Elles révèlent également l'attention d'un chercheur à l'actualité scientifique de son domaine. Plus fondamentalement, c'est la continuité constitutive de toute élaboration scientifique que donne à voir le réinvestissement textuel, cette continuité pouvant ou non être montrée aux étudiants dans l'enseignement final.

9 Diversement attesté selon les fonds d'archives des chercheurs, le phénomène de réinvestissement textuel est une reconstruction de la part de l'observateur, qui doit reconstituer le « déjà-là » de l'écrit à partir d'indices qui sont de deux ordres : matériel et discursif. Les indices d'ordre matériel englobent toutes les informations codicologiques exploitables à partir de l'étude du support (nature, couleur et format du papier, traces éventuelles de trombones...), de l'encre utilisée, du tracé ou de la disposition de l'écrit. Les indices discursifs intègrent à la fois les manifestations linguistiques de renvois à des écrits antérieurs et les phénomènes d'intertextualité non signalés comme tels. Le réinvestissement d'un texte antérieur avec les outils propres à la langue rencontre la problématique générale du dialogisme, ou plus précisément de l'« autodialogisme » ou du « dialogisme intradiscursif ». L'interaction du discours avec ses discours antérieurs peut prendre différentes formes, explicites ou implicites: allusion, renvoi explicite, autocitation, discours rapporté marqué et non marqué, ainsi que le vaste domaine de la " réécriture » entendue comme le fait de " "revenir sur un déjà écrit" pour le reprendre, le retravailler peut-être, par une série d'approximations successives, au cours de l'élaboration d'un texte. » (Grésillon in C. Boré et C. Doquet-Lacoste, 2004, p. 9)

10 Ces deux grands registres d'indices - matériels et linguistiques - dessinent les deux grands types de réinvestissement textuel identifiables dans les fonds d'archives des enseignants-chercheurs ; deux types qui correspondent à deux acceptions du «texte » réinvesti : texte comme objet matériel et texte comme objet discursif. Dans la limite de cette étude, nous nous concentrerons sur le premier type de réinvestissement, moins étudié par les linguistes et les généticiens que le réinvestissement d'ordre discursif.

\section{Aspects matériels du réinvestissement textuel}

Les opérations d'écriture définies par la critique génétique, ajout, suppression et déplacement, ne comportent pas de spécification matérielle : insérer une phrase en l'inscrivant en marge ou en interligne, ou bien en procédant par collage, ne saurait pourtant être décrit comme strictement équivalent, ni du point de vue de l'action, ni du point de vue de résultat - et il en va de même pour le découpage, qui agit autrement que la rature. (Bustarret, 2011, p. 354)

11 Le découpage et le collage, signalés par C. Bustarret, mais aussi le déplacement de feuillets, sont à inclure dans un ensemble d'opérations matérielles qui témoignent du réinvestissement textuel en tant que processus d'écriture. Le support d'écriture conserve en effet, dans tous ces cas, la mémoire de textes antérieurs dans leurs dimensions discursives et matérielles, tout en s'insérant dans la structure organisationnelle d'un 
nouveau texte, que ce soit à l'échelle d'un feuillet ou à celle d'un ensemble de feuillets constituant un cours.

\section{Déplacer : l'exemple de Charles Bally}

12 La réutilisation telles quelles de notes de cours antérieures semble, dans la préparation d'un enseignement, une pratique parfaitement banale ${ }^{7}$. Pour autant si ce geste ne s'accompagne pas d'inscription nouvelle ou, comme dans le cas du découpage-collage, d'altération du support, elle risque de passer inaperçue. À moins d'un contraste codicologique (différence de papier, d'encre ou d'écriture avec les feuillets qui l'entourent), le geste de déplacement d'un écrit antérieur dans un nouveau dossier ne laisse pas de trace. Cette pratique peut néanmoins être observée dans les notes de cours de C. Bally grâce à son habitude de signaler la réutilisation de ses papiers.

Nous nous intéresserons ici aux cours de linguistique générale qu'il donne à l'Université de Genève entre 1913 et $1939^{\circ}$. Au cours de cette période, comme le note C. Forel (2008, p.107), «Bally réutilisait ses notes pour des enseignements ultérieurs mais il semble n'avoir jamais donné tout à fait le même cours ». Au début de chaque liasse de feuillets préparatoires à un cours, le linguiste insère a posteriori un feuillet dactylographié indiquant les remaniements dont les notes ont fait l'objet. Pour le cours de 1915-1916, par exemple, on trouve :

Figure 1. BGE, Ms. fr. 51025 , f. 1

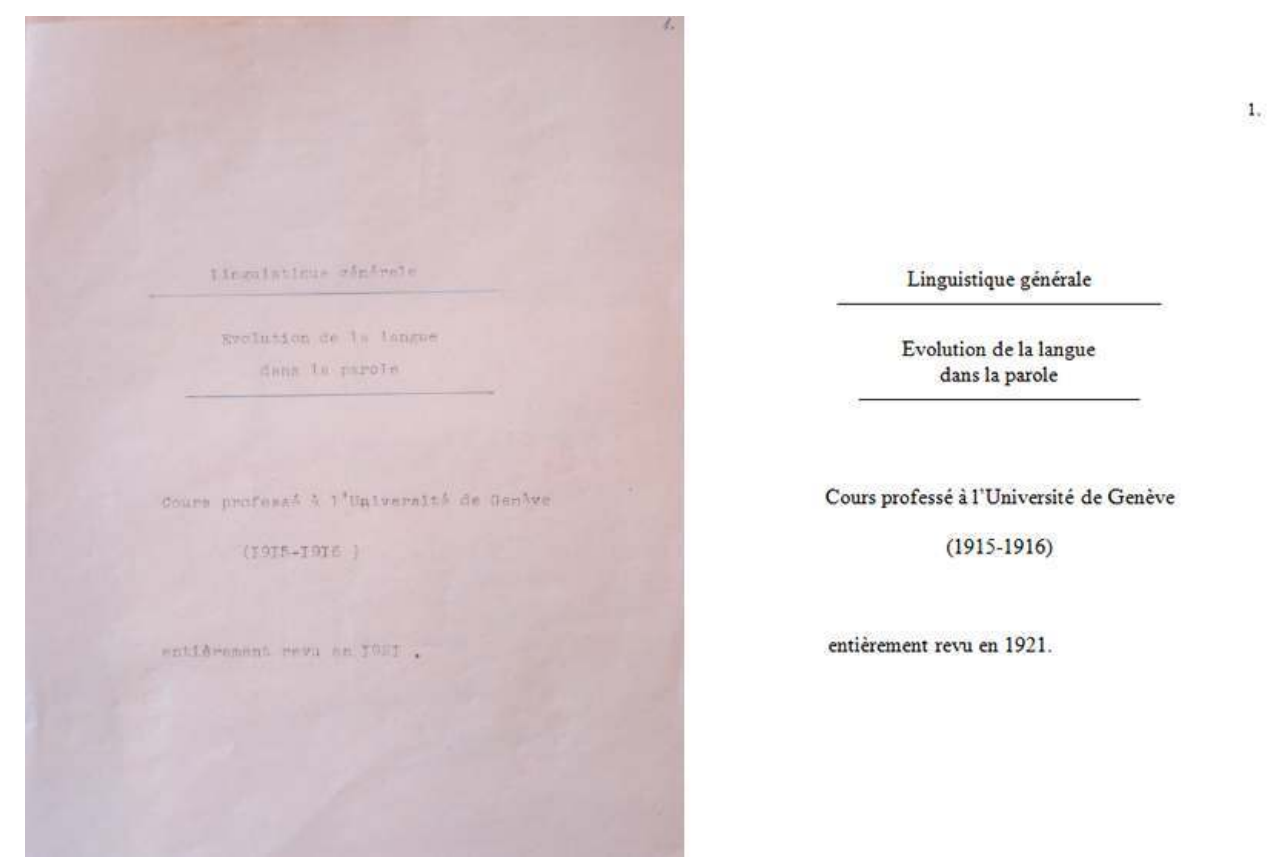

Pour celui du semestre d'été 1926, dont les papiers sont plusieurs fois repris, il indique : 
Figure 2. BGE, Ms. fr. 5044, f. 1

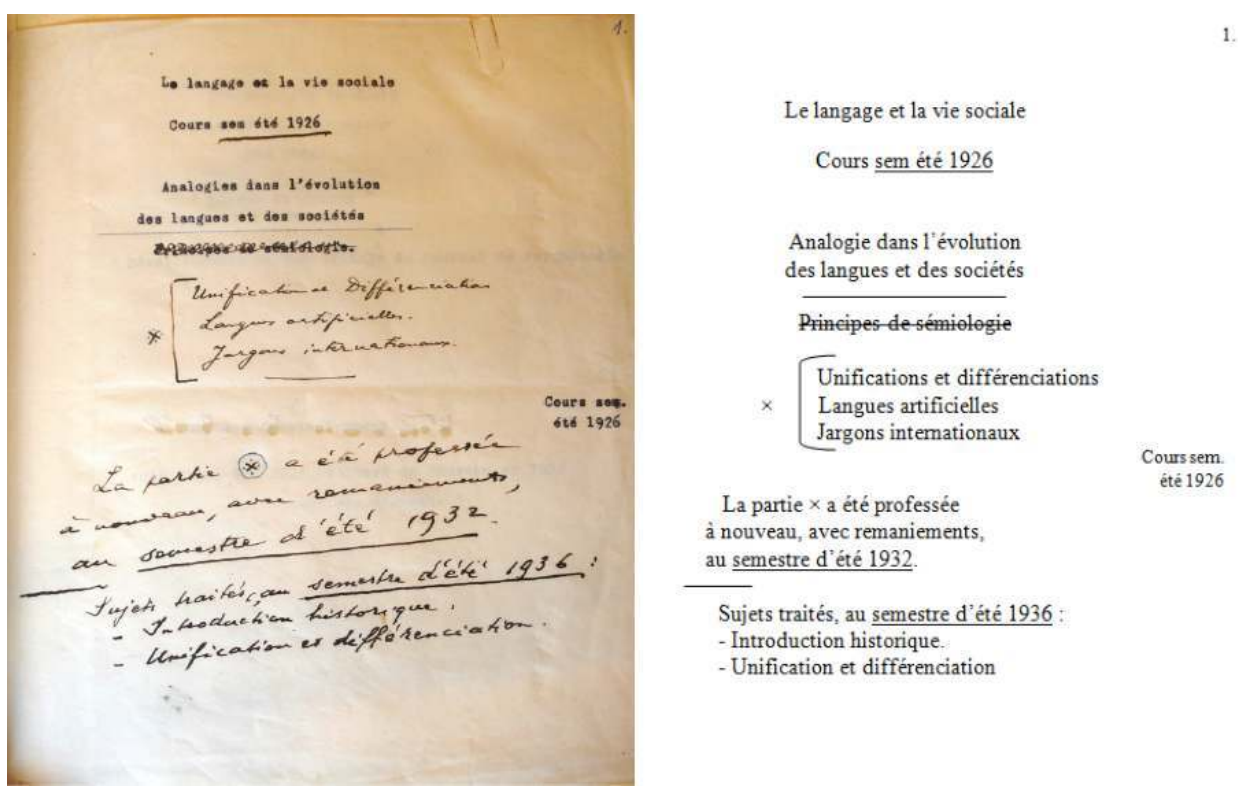

Dans le détail de ses notes, C. Bally s'efforce d'indiquer chaque réutilisation dont ses papiers ont fait l'objet, tout particulièrement le déplacement des matériaux écrits. Le manuscrit du cours de 1918-1919 repris en 1921 (BGE, Ms. fr. 5027, f. 1-38) est exemplaire de cette pratique. Le dossier des notes préparatoires de ce cours, tel qu'il est conservé aujourd'hui à la Bibliothèque de Genève, est exceptionnellement bref - il ne compte qu'une quarantaine de feuillets - car la majorité des feuillets qui le constituaient ont été déplacés par le linguiste pour la préparation de cours ultérieurs. À l'endroit des feuillets déplacés, $C$. Bally a glissé des feuillets fantômes qui gardent la trace de ces déplacements. Ces feuillets sont dactylographiés et se présentent toujours de la même façon: ils comportent le titre du passage ou du chapitre déplacé, le lieu où il a été déplacé et éventuellement le titre de son nouvel emplacement. Dans le corps du dossier du cours de 1918-1919, on trouve ainsi une dizaine de ces feuillets fantômes portant les indications suivantes :

Prestige du progrès. Cette partie a été versée dans le Cours été 1924 (chapitre «préjugés sur la langue maternelle et la supériorité de diverses langues ».) (BGE, Ms. fr. 5027, f. 16)

Valeurs et signes linguistiques. Valeurs et symboles sociaux. Voir cours été 1925. ( Ibid. f. 20)

Dialectes et langues spéciales. Ce chapitre a été versé dans le Cours « langage et vie sociale » 1922-1923. (Ibid. f. 21)

Types sociaux et linguistiques. [...] (Ce chapitre a été fondu dans deux chapitres du nouveau cours. a) langue indice d'ethnisme, b) classification des l. et des s. (Ibid. f. 22)

Évolution comparée avec la synchronie. Ce chapitre a été incorporé dans le « cours $1924 »$ (Ibid. f. 23)

Linguistique génétique. Sémiologie générale. Voir fin du cahier - où ces notes ont été transportées sans changement (Ibid. f. 24)

On notera les variations dans la façon dont le linguiste désigne la réutilisation de ces papiers: "a été versé », "a été fondu», "a été incorporé», "a été déplacé »... Ces variations reflètent la diversité des modalités du réinvestissement textuel : si certains groupes de feuillets ont été simplement déplacés, la plupart du temps la manipulation 
matérielle s'accompagne aussi d'un geste de réécriture. Insérés dans de nouveaux ensembles, les feuillets ne gardent pas nécessairement leur cohérence et leur succession premières. C. Bally rédige parfois de nouveaux feuillets qu'il intercale entre eux. Ainsi une page qui correspondait à un chapitre dans un cours devient, après le déplacement, la partie d'un nouveau développement plus large. Les différentes numérotations qui cohabitent sur les feuillets permettent de suivre ces phénomènes d'expansion ou de réduction d'un cours à l'autre.

Prenons un exemple de ces déplacements de feuillets couplés à une réécriture. En 1933, C. Bally donne un cours de linguistique générale sociologique qu'il intitule "Langage et vie sociale ». Il reprend pour cela les notes, majoritairement dactylographiées, d'un cours donné en $1923^{9}$. Ces notes font, à cette occasion, l'objet de réaménagements importants. Ainsi en va-t-il pour le passage consacré aux « interdictions linguistiques ", c'est-à-dire aux «tabous». Dans les notes de 1923, C. Bally distingue trois types d'interdictions: relatives aux choses, aux personnes et à la langue elle-même :

Figure 3. BGE, Ms. fr. 5027, f. 188 (détail)

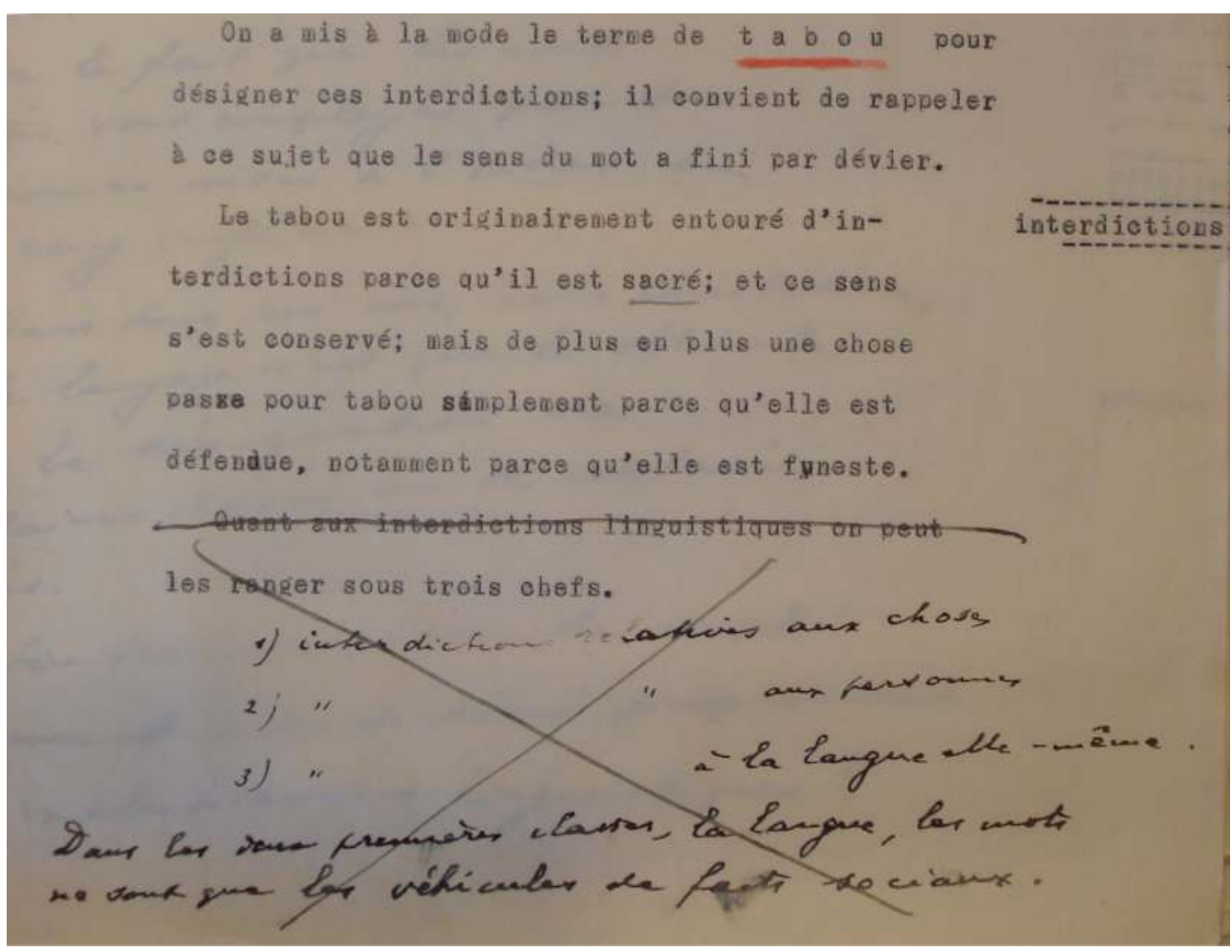

En 1933, C. Bally, on le voit, barre ce passage et lui substitue sur un feuillet libre, une partition à quatre entrées : 

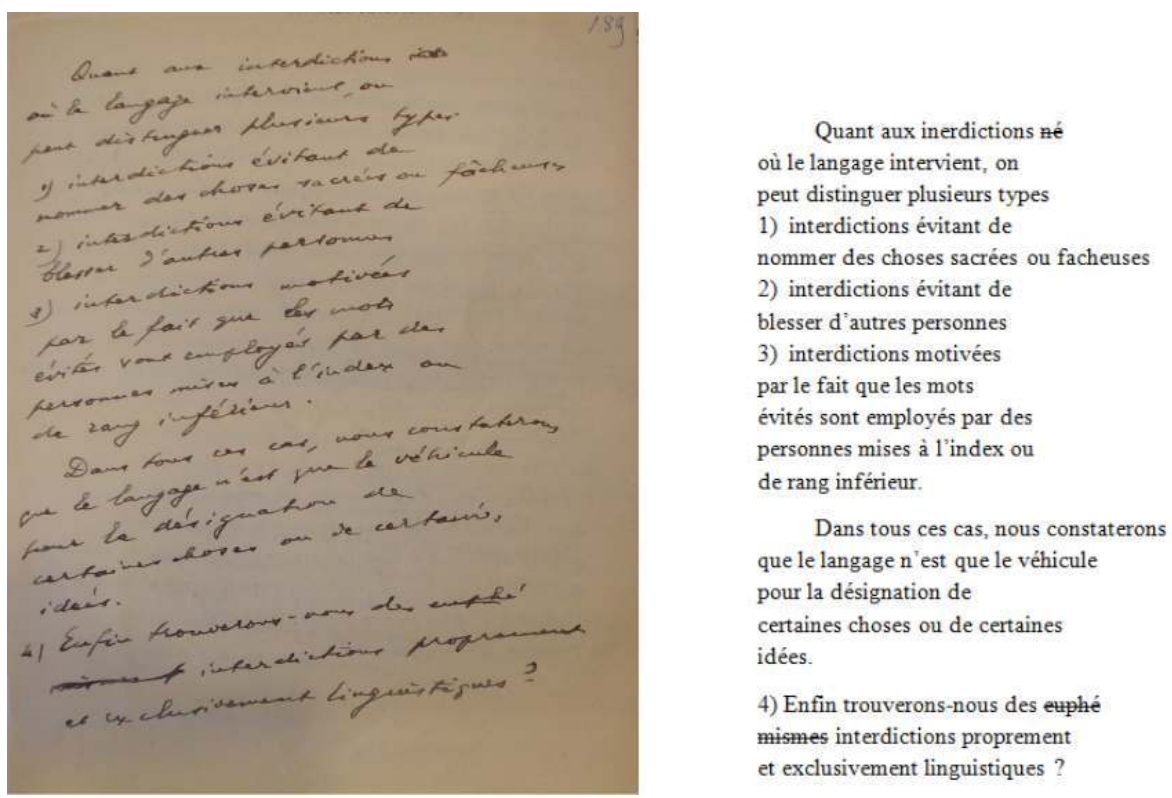

La modification du plan correspond à un changement de fond: l'existence de tabous exclusivement linguistiques présentée de façon assertive en 1923 est interrogée dix ans plus tard. Elle correspond également à une extension considérable du champ des tabous linguistiques liés aux relations sociales, question que C. Bally a particulièrement travaillée au cours des années 20 . La modification de cette typologie implique un travail sur les notes du cours de 1923 chargées de la développer pour les étudiants. D'une dizaine de pages en 1923, le développement passe à une vingtaine en 1933. De nouveaux feuillets sont intercalés autant que les anciens feuillets sont réaménagés. L'ancienne deuxième partie, par exemple, est scindée en deux (les points 2) et 3) de la nouvelle typologie), ce qui se traduit par une modification de l'ancienne numérotation (v. figure 5) et par l'introduction d'un nouveau passage sur les tabous liés aux relations sociales contrepartie de ce que C. Bally appelle «la mode»- qui n'existait pas en 1923. Ce sont donc bien des choix théoriques et didactiques qui président au déplacement et au réaménagement des notes.

Figure 5. BGE, Ms. fr. 5027, f. 194 (détail)

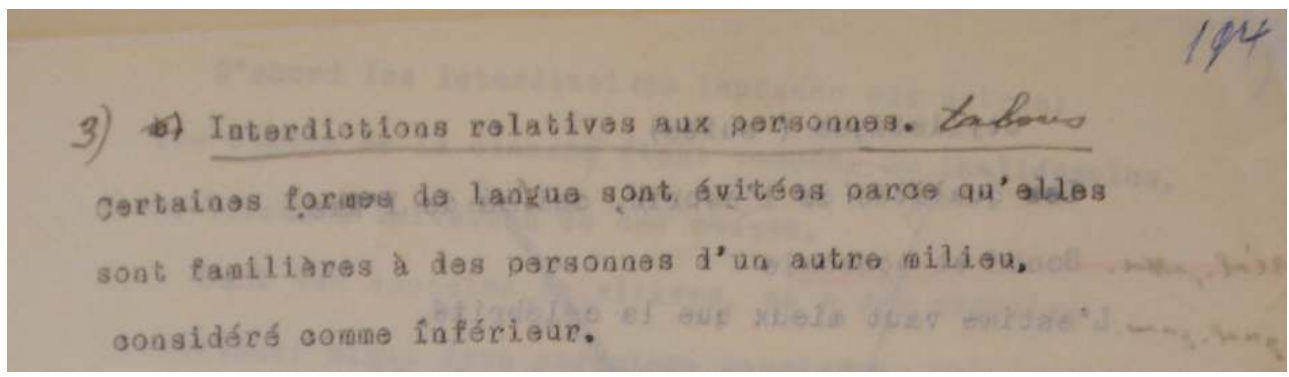

L'habitude qu'a C. Bally de déplacer ses feuillets d'un dossier à l'autre au fur à mesure de la préparation de ses cours a une conséquence pratique : elle rend définitivement perdue la structure originelle des cours, chaque feuillet déplacé pouvant faire l'objet de nouveaux déplacements dans un cours ultérieur sans que les indications de C. Bally soient suffisantes pour reconstruire l'ordre origine ${ }^{10}$. Les archives conservent la disposition 
finale des papiers du linguiste au terme des processus de déplacement et de réemploi. Les dossiers des cours les plus anciens - comme celui de 1918-1919 - sont pour ainsi dire vidés de leur substance; seuls ceux qui se situent au bout du processus de réinvestissement textuel sont complets. En définitive, les notes de cours de C. Bally sont à l'image de la langue telle que la décrit son maître F. de Saussure: une robe rapiécée de sa propre étoffe.

\section{Découper et coller pour recomposer : l'exemple de Ferdinand Brunot}

F. Brunot réutilise fréquemment ses propres écrits, qu'il découpe et qu'il colle sur de nouveaux feuillets. Ce phénomène apparaît en particulier dans les notes de son cours d'histoire de la langue française, professé en Sorbonne entre 1900 et 1934. On prendra ici un exemple représentatif de ce type de réinvestissement textuel.

Dans une leçon intitulée «Latin écrit et latin parlé », F. Brunot aborde les conséquences sur la formation du français de la différenciation entre les variétés du latin. Un feuillet, numéroté « 8 » en haut à droite ${ }^{11}$, traite des erreurs en recherche étymologique. Ce feuillet comporte trois fragments collés ainsi que des segments linguistiques écrits directement sur la feuille, qui complètent et relient les fragments. Ainsi, les deux premiers fragments, accolés verticalement, forment une phrase unique, enchaînée au troisième fragment :

Figure 6a. Bibliothèque de l'Institut de France, Fonds F. Brunot, Ms 7783, « Latin écrit, latin parlé ». Reproduction photographique

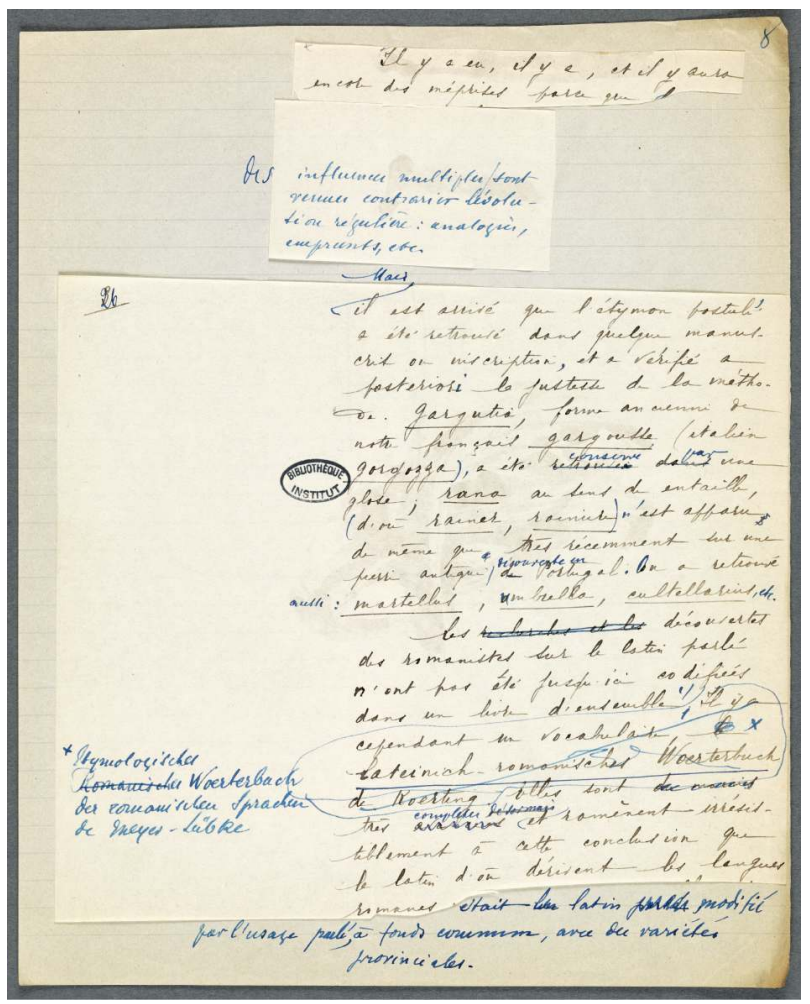


Figure 6b. Bibliothèque de l'Institut de France, Fonds F. Brunot, Ms 7783, « Latin écrit, latin parlé ». Transcription

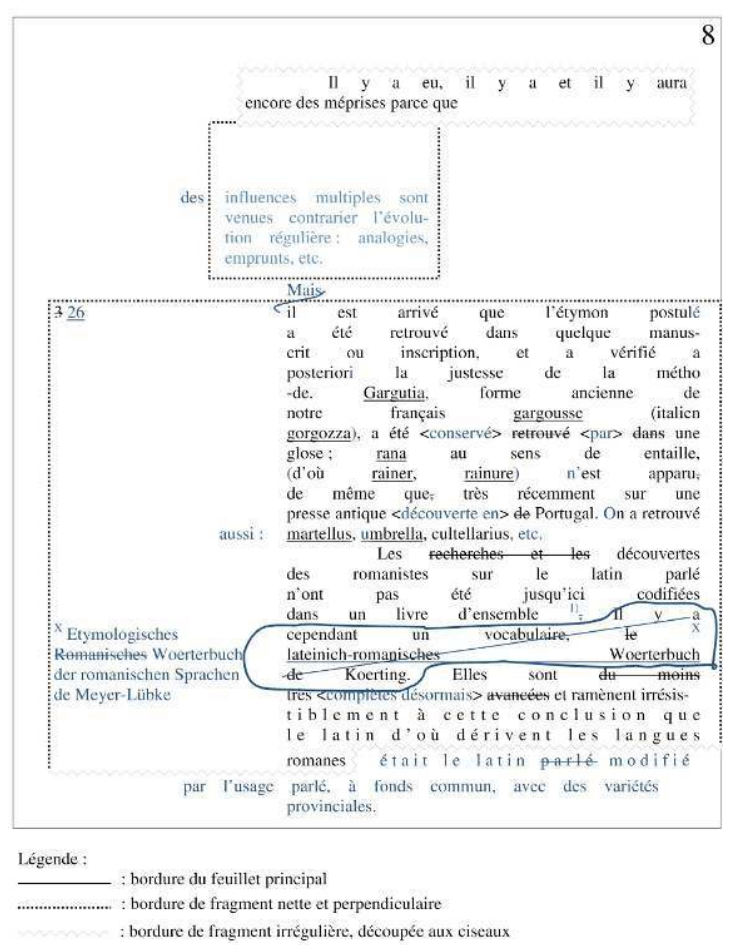

Plutôt que de réécrire entièrement ce passage, F. Brunot choisit de réutiliser des extraits déjà rédigés séparément. La similitude de l'encre, du papier et de l'écriture laisse penser que le premier fragment provient du même texte initial que le troisième, sans qu'il soit possible de déterminer s'ils se trouvaient sur le même feuillet ou non. Ces deux fragments sont également à rapprocher sur le plan du contenu dans la mesure où ils traitent tous les deux du même problème métalinguistique, celui de la reconstruction en recherche étymologique. F. Brunot signale, dans le premier fragment collé, que cette méthode peut donner lieu à des erreurs, des "méprises ", avant de donner dans le troisième fragment des exemples de cas où des formes reconstruites ont été attestées par la suite. Le lien logique qui relie ces deux éléments est matérialisé par l'ajout du mot «mais» qui introduit le troisième fragment, à l'encre bleue sur la feuille principale. Pour autant, malgré la connexion que F. Brunot conserve ou, du moins, établit entre ces deux fragments, ils restent séparés par un autre, de nature différente.

Concernant le second fragment, étant donné son format, qui correspond à celui de nombreuses petites fiches dont on trouve plusieurs jeux dans le fonds F. Brunot, il est vraisemblable que ce soit l'une de ces fiches qui ait été directement insérée, par collage, entre les deux autres fragments ${ }^{12}$. Bien que contraignante par sa fonction synthétique et son format réduit, la fiche est déjà complète sur le plan sémantique et syntaxique; c'est ce qui lui permet de s'insérer au sein du nouveau texte sans autre modification que l'ajout de l'article "des", qui sert à assurer l'enchaînement avec le fragment précédent, constituant ainsi une phrase unique.

Ce second fragment diffère également des deux autres par son contenu discursif. Introduit par le "parce que » qui clôt le premier fragment, il décrit la cause des "méprises» en recherche étymologique; F. Brunot cite ainsi des phénomènes qui 
relèvent d'une approche diachronique de la langue, tels que les emprunts ou les analogies. Le découpage et le collage à cet endroit suggèrent justement que la cause que F. Brunot choisit d'évoquer dans son cours a changé par rapport à la rédaction initiale du texte dont sont tirés les premier et troisième fragments, sans qu'il soit possible de déterminer si cela relève d'une modification de fond - une cause différente - ou de forme - une nouvelle formulation. Toujours est-il que F. Brunot met à jour les connaissances qu'il transmet à ses étudiants, ce qui passe par la mise en relation de plusieurs domaines de connaissance : la linguistique historique et l'évolution interne, régulière, des langues ; l'histoire externe de la langue et les «influences » qui "contrarient» la régularité de cette évolution; et, enfin, les méthodes et les résultats du travail métalinguistique qui permettent d'identifier ces phénomènes. De ce point de vue, ce fragment est à rapprocher de l'ajout à l'encre bleue qui clôt le feuillet: celui-ci apporte des informations véritablement nouvelles qui complètent le dernier fragment collé, non seulement sur le plan syntaxique, mais aussi sémantique. F. Brunot en arrive alors à la conclusion de son raisonnement sur la recherche étymologique, qui n'est visiblement plus la même qu'à l'époque de la rédaction du troisième fragment collé.

Le travail de découpage et de collage révèle donc, dans un premier temps, la permanence des problèmes abordés, d'une part, et l'accrétion des savoirs, qui autorise la conservation des exemples, d'autre part. Cependant, il ne s'agit pas ici de former des romanistes chevronnés, mais des professeurs de français qui, dans les établissements secondaires, enseigneront aussi bien le français que le latin. C'est pourquoi F. Brunot ne donne pas les références exactes des articles où sont exposées les recherches d'où il tire ses informations : l'exploitation des exemples et les conclusions qu'il en tire priment sur l'exactitude bibliographique. D'ailleurs, la seule référence précise, celle de W. MeyerLübke, est erronée. En effet, F. Brunot renvoie à un ouvrage de W. Meyer-Lübke, qu'il intitule "Etymologisches Romanisches Wörterbuch der romanischen Sprachen»; or le titre de l'ouvrage de W. Meyer-Lübke, paru en 1911, est Romanisches etymologisches Wörterbuch. Le titre inscrit sur le feuillet est celui d'un ouvrage de F. Diez, paru en 1853. Il y a manifestement erreur dans l'association du titre et de l'auteur, ce que F. Brunot n'a pas pris la peine de vérifier ou de corriger.

En deuxième analyse, le découpage-collage est également un moyen de mettre à jour des connaissances factuelles ainsi que de relier entre eux différents types de savoirs, complémentaires dans le cadre de la formation de futurs enseignants. Le manuscrit donne ainsi à voir la fabrique du savoir enseigné, à la croisée de plusieurs temporalités : celle de l'élaboration d'un savoir disciplinaire en linguistique historique ; celle de la constitution du savoir, des références et de la pensée de F. Brunot; et celle de la construction du cours, en différentes phases de travail. On peut repérer au moins trois phases d'écriture différentes: une phase de rédaction du texte d'où sont tirés le premier et le troisième fragment; une phase de confection de fiches, dont celle qui constitue le deuxième fragment ; une phase d'écriture sur le nouveau feuillet, afin de relier et de compléter les fragments collés. Cependant, hors de ces phases d'écriture, a nécessairement eu lieu une relecture du texte d'où sont tirés deux des fragments collés, d'une part, et des fiches d'où provient le dernier fragment, d'autre part. De plus, il a fallu sélectionner les fragments et les organiser, avant de faire le travail de mise en page, centré autour du découpagecollage.

Dans les manuscrits d'enseignement de F. Brunot, nombreux sont les feuillets comme celui-ci, qui, recomposés à partir d'anciens fragments, commencent par une nouvelle 
phrase et même fréquemment par un nouveau paragraphe. Chez lui, le réinvestissement textuel, quand il prend la forme d'un découpage-collage, tend à faire du feuillet sur lequel est composé le nouveau texte une unité autonome. La recomposition n'est donc pas une simple correction, c'est une réécriture complète. Le réinvestissement textuel s'appuie ici sur la complémentarité des types d'écrits, dans laquelle la décomposition d'ensembles préexistant sous forme de textes rédigés ou de fiches synthétiques, mais qui ne peuvent être repris tels quels, ouvre la possibilité de construire un nouveau texte, qui corresponde aux conceptions que F. Brunot veut enseigner au moment de la réécriture.

\section{Découper et coller pour insérer : l'exemple d'Arsène Darmesteter}

Comme F. Brunot, A. Darmesteter s'intéresse à la transition du latin aux langues romanes en général et au français en particulier. Il donne ainsi un cours sur le " latin populaire ", probablement à l'EPHE dans le cadre de la «conférence des langues romanes " ${ }^{13}$. L'ensemble comprend vingt feuillets, dont beaucoup ont été numérotés à plusieurs reprises par A. Darmesteter lui-même, ce qui témoigne d'une réorganisation, si ce n'est d'un réemploi. A. Darmesteter semble donc, comme C. Bally, déplacer ses papiers sans qu'il soit possible de déterminer dans quel ordre ils étaient originellement agencés. En revanche, l'ordre dans lequel sont actuellement conservés les feuillets semble assez fréquemment briser la linéarité du texte. En effet, contrairement à ce qu'on observe chez F. Brunot, chaque feuillet ne constitue pas une unité autonome par rapport aux autres.

Alors qu'A. Darmesteter n'hésite généralement pas à rayer des paragraphes entiers et à en réécrire une nouvelle version ou à ajouter des paragraphes, entre les lignes barrées ou au verso du même feuillet - ce qui en rend la lecture extrêmement difficile -, le vingtième ${ }^{14}$ et dernier feuillet de l'ensemble présente un cas de collage. Il est composé de deux parties, de surfaces environ égales. La partie supérieure est écrite à l'encre noire sur du papier ligné, différent des autres feuillets du même cours. Cette partie provient vraisemblablement d'une feuille découpée à la main horizontalement puis collée sur le feuillet principal. À l'inverse, l'aspect matériel de la partie inférieure du feuillet correspond en tout point à celui des feuillets précédents. 
Figure 7a. Bibliothèque Interuniversitaire de la Sorbonne, Papiers Arsène Darmesteter, II Ms AUC 41. Reproduction photographique

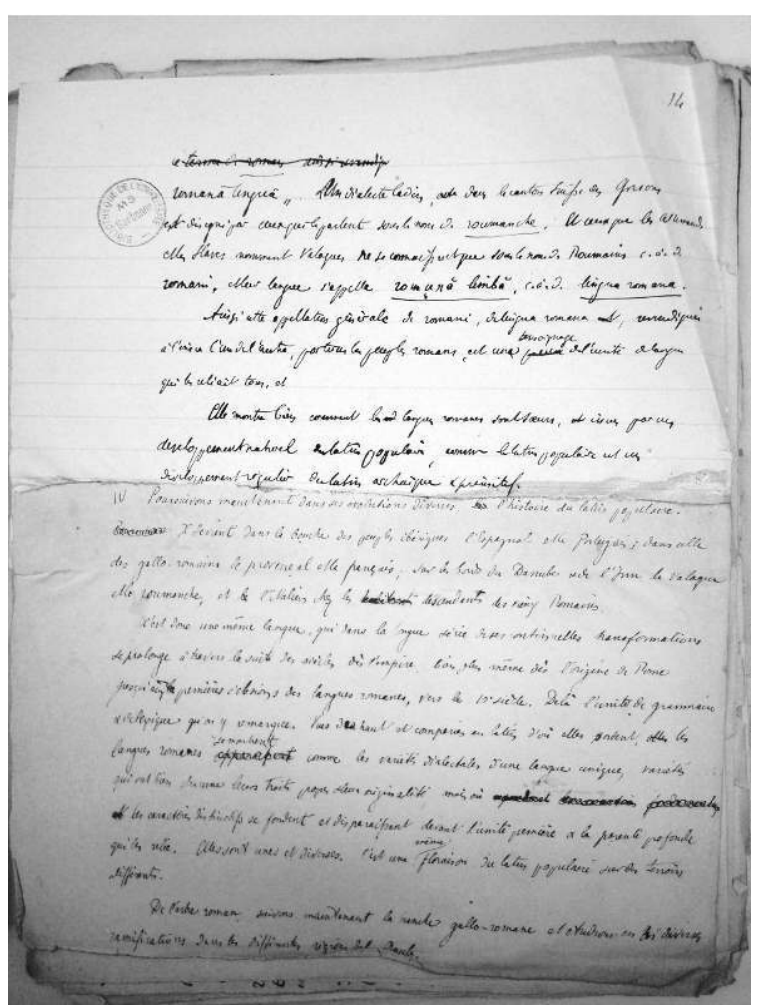

Figure 7b. Bibliothèque Interuniversitaire de la Sorbonne, Papiers Arsène Darmesteter, II Ms AUC 41. Transcription

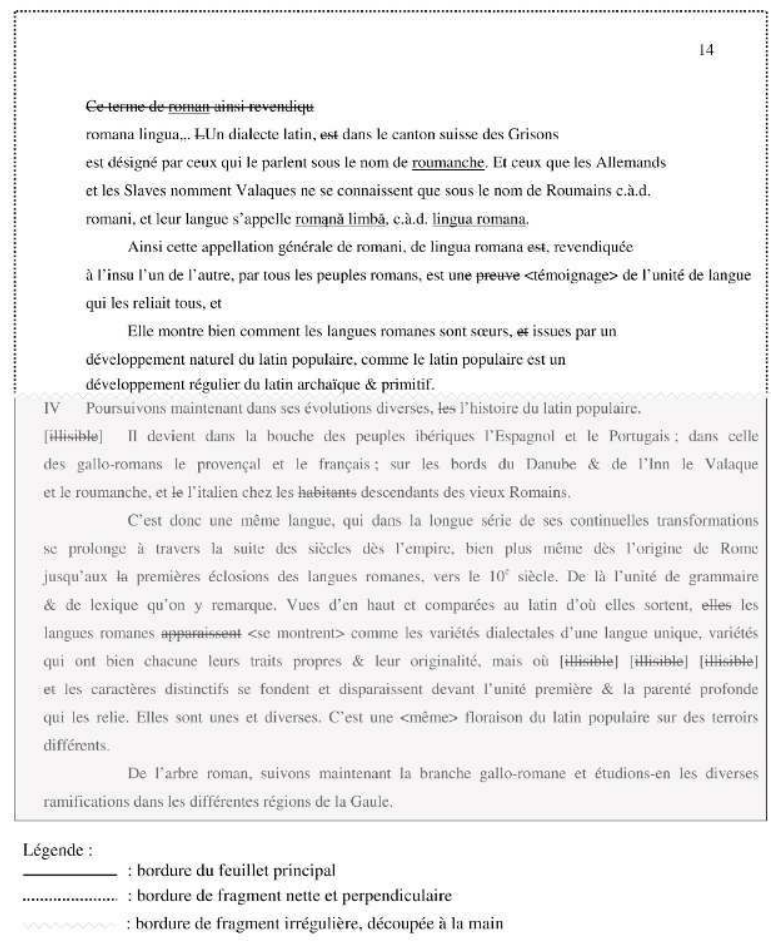


31 Le texte écrit sur le fragment collé a été rédigé avant le découpage : les jambes des $p$ du mot « développement ", à la dernière ligne, sont coupées. Cependant, le fragment collé ne marque pas le début d'une nouvelle phrase ni d'un nouveau paragraphe ; à l'opposé, les premiers mots voulus par A. Darmesteter, "romana lingua » suivis de guillemets fermants, terminent une citation en latin débutée sur le feuillet précédent, où la citation a été ajoutée avec la même encre que celle utilisée sur le fragment collé. A. Darmesteter avait initialement prévu de faire figurer toute la citation sur le feuillet précédent: les mots « romana lingua » et les guillemets fermants s'y trouvent, barrés, sur la dernière ligne du texte principal, juste avant les notes de bas de page. Ceci est confirmé par la toute première ligne du fragment collé, où l'on peut lire un début de phrase, abandonnée en cours et barrée : «Ce terme de roman ainsi revendiqu ». A. Darmesteter cherche donc à rendre le fragment collé indissociable de ce qui précède.

32 Par ailleurs, la première ligne du texte qui suit le fragment collé, et clôt le cours, est numérotée «IV » et débute un nouveau paragraphe. La partie recouverte par le fragment collé formait donc un ensemble de paragraphes qu'A. Darmesteter a choisi de remplacer par un nouveau passage, tout en conservant à la fois ce qui précède et ce qui suit. Ainsi le fragment collé a-t-il été écrit directement en vue de remplacer la partie qu'il recouvre.

Du point de vue du contenu, ce fragment collé comporte, dans un premier temps, des exemples qui illustrent le phénomène qui intéresse ici A. Darmesteter, à savoir le fait que plusieurs langues portent des noms équivalents à lingua romana; puis, dans un second temps, suivent des conclusions tirées de ce constat, introduites par " ainsi ». Ce feuillet vient terminer un ensemble qui comprend les feuillets 16 à 20, dans lequel A. Darmesteter présente les limites géographiques de la Romania; or, cet extrait est en fait une réécriture des feuillets 7 à 10. Dans cette seconde version, A. Darmesteter conserve entièrement les citations, les références ainsi que certaines formulations, de même que l'organisation des paragraphes de la première version. En revanche, à la fin de la première version, A. Darmesteter ne faisait qu'annoncer ce passage sur la dénomination lingua romana :

Mais nous verrons qu'en général les divers peuples romans attribuèrent exclusivement ce nom [i.e. lingua romana] à leur langue propre, n'ayant pas conscience de l'unité primitive qui les avait jadis reliés entre eux, et dont ce terme cependant était un souvenir assez net encore.

Dans la seconde version, ce thème occupe une grande partie du feuillet 19 et la partie supérieure du feuillet 20 , celle qui a été découpée et collée. Le contenu des feuillets 7 à 10 est donc réinvesti, peut-être en partie pour des questions pratiques de lisibilité, ces feuillets comportant de nombreux ajouts et ratures; c'est surtout l'occasion d'approfondir un point qu'A. Darmesteter souhaitait déjà aborder mais qui n'avait pas encore fait l'objet d'un réel traitement. Il s'agit de replacer les langues romanes dans un continuum par rapport au latin populaire et, de façon plus lointaine, au latin archaïque. Néanmoins, la dernière phrase du fragment est extrêmement similaire à une autre, qu'on peut lire sur le feuillet 5. Comparons :

Ainsi cette appellation générale de romani, de lingua romana, revendiquée à l'insu l'un de l'autre, par tous les peuples romans, est un témoignage de l'unité de langue qui les reliait tous, et elle montre bien comment les langues romanes sont sœurs, issues par un développement naturel du latin populaire, comme le latin populaire est un développement régulier du latin archaïque et primitif. (f. 20$)^{15}$

Résumons maintenant les remarques diverses qui précèdent. Tous ces exemples prouvent que les traits propres aux langues romanes appartiennent au latin 
populaire : esprit d'analyse, recours à des constructions plus simples, plus claires, large développement donné à la dérivation. Enfin le lexique des L[angues] $R$ [omanes] reproduit non le lexique du lat[in] classique plus ou moins corrompu, mais celui du lat[in] vulgaire. Tous ces faits justifient donc la théorie qui voit dans les $L$ [angues] $R$ [omanes] le développement naturel et spontané du lat[in] populaire comme celuici est le développement du lat [in] archaïque et primitif. (f. 5) ${ }^{16}$

Même si l'idée que les langues romanes sont «sœurs" s'appuie spécifiquement sur l'argument développé dans les feuillets 19 et 20, la conclusion à laquelle arrive A. Darmesteter à la fin du fragment collé sur ce dernier feuillet est quasiment identique à celle rédigée au terme d'une partie sur les caractéristiques du latin populaire. Cette idée, qu'A. Darmesteter semble vouloir absolument transmettre à ses étudiants, est le fil rouge de l'ensemble du discours, malgré les ruptures dans la linéarité. Il est, par conséquent, capital de la formuler précisément, d'une part, et de la faire apparaitre comme la conclusion logique du raisonnement didactisé, d'autre part ; ceci se traduit par une forme de réinvestissement discursif. Ainsi, les aspects matériels et discursifs du réinvestissement textuel ne concordent pas parfaitement : le fragment collé, plus récent que le reste des notes de cours, se compose, sur le plan textuel, d'une partie nouvelle, où sont donnés des exemples d'appellations de langues romanes, et d'un passage réinvesti. À l'inverse du feuillet de F. Brunot précédemment analysé, le réinvestissement textuel, sur le plan matériel, ne se situe pas du côté du fragment collé, bien que ce dernier en soit en partie le support sur le plan linguistique et discursif. Le découpage-collage participe donc d'une modification de la trame du raisonnement, mais aboutit à une conclusion qui, elle, est « déjà-là ».

\section{Conclusions}

Pour les chercheurs d'aujourd'hui, les archives des enseignants-chercheurs d'hier conservent la mémoire de méthodes de travail visant à concilier deux exigences de l'enseignement supérieur : l'exactitude scientifique et la clarté didactique. Cette double exigence s'obtient au prix d'un travail élaboré, les opérations qui interviennent dans la réutilisation d'écrits antérieurs révélant à la fois leur diversité et leur complexité. Dans la description des processus d'écriture qui s'appuient sur du " déjà écrit », il convient donc de prendre en compte les indices d'ordre sémiotique et les manipulations matérielles qui s'imbriquent dans les réécritures. Les écrits didactiques de C. Bally, de F. Brunot et d'A. Darmesteter se signalent, au cours de leur élaboration, par une grande instabilité matérielle: soumis à des opérations de déplacement, de découpage et de collage, ces documents gardent à la fois la trace d'écrits antérieurs et de différentes phases de lecture, d'écriture, de relecture, et de réécriture. Loin d'être une solution de facilité - une manière simple de refaire chaque année le même cours - le réinvestissement textuel apparait précisément chez ces trois linguistes comme un moyen d'éviter l'écueil de la répétition, à travers des opérations de sélection, d'agencement et de recomposition.

Dans ce travail, les hésitations sont nombreuses, mais une constante reste le rôle essentiel du « déjà-là ». Les unités qui composent les cours, qu'elles se présentent sous forme de feuillets individuels ou d'ensembles de feuillets, de paragraphes ou de parties entières, témoignent d'une forte autonomie les unes par rapport aux autres : c'est ce qui permet de les remplacer ou de les déplacer aisément, mais aussi de les associer entre elles, gestes d'écriture communs à C. Bally, F. Brunot et A. Darmesteter. Pour ces scripteurs experts, spécialistes reconnus dans leur domaine, c'est la mise à jour des savoirs enseignés, mais 
aussi et surtout l'organisation logique, toujours à améliorer et à expliciter, qui semblent présider à l'écriture d'un cours. Écrire pour enseigner apparaît alors comme une manière de mettre en cohérence et de relier entre eux, de façon très concrète, des éléments issus de phases de travail distinctes afin de créer de nouveaux ensembles de connaissances qui feront l'objet d'un enseignement. Le savoir ainsi construit est donc le fruit d'un véritable travail d'élaboration qui ne se réduit pas à une simple reformulation de connaissances préexistantes. L'enjeu est bien de présenter aux étudiants de manière claire un état de connaissances qui paraisse stable et actualisé : pour diffuser un savoir "disponible », il n'est pas nécessaire de montrer le travail de «mise à disposition». Se trouve donc en cause la validité de l'affirmation selon laquelle «ce qui caractérise l'enseignement de niveau supérieur, c'est qu'il transmet directement le savoir » (Chervel, 1988). En effet, la didactisation, «au confluent des processus de diffusion et d'application " (Chiss et al., 2012), confère aux notions de réemploi, de reformulation ou d'autocitation une dimension qui dépasse la simple vulgarisation ou la simplification: il s'agit à la fois d'organiser des savoirs préexistants de manière à les rendre enseignables, et de construire, de façon plus ou moins explicite, un rapport de légitimation réciproque entre le travail de recherche et celui d'enseignement.

\section{BIBLIOGRAPHIE}

BALLY, C. (1930). La crise du français : notre langue maternelle à l'école. Neuchâtel : Delachaux/Niestlé. - (1935). Le langage et la vie. Genève : Droz.

BARBEROUSSE, A. \& PINON, L. (dirs) (2003). Genesis. Manuscrits, Recherche, Invention 20 : « L'écriture scientifique $»$.

BENVENISTE, É. (1969). Le vocabulaire des institutions indo-européennes. Paris : Minuit.

BERgounioux, G. (1984). « La science du langage en France de 1870 à 1885 : du marché civil au marché étatique ». Langue française 63 (1), p. 7-41.

- (1990). « L'enseignement de la linguistique et la philologie en France au XIX ${ }^{e}$ siècle d'après les affiches de cours des facultés de lettres (1845-1897) ». Archives et documents de la S.H.E.S.L. 2, p. 1-105. - (1998). « Science et institution : la linguistique et l'université en France (1865-1945) ». Langue française 117 (1), p. 22-35.

BERT, J.-F. (2014). Qu'est-ce qu'une archive de chercheur. Marseille : OpenEdition Press. En ligne : http://books.openedition.org/oep/438.

BORÉ, C. \& DOQUET-LACOSTE, C. (2004). « La réécriture, questions théoriques ». Le français aujourd'hui 144 (1), p. 9-17.

BRUNOT, F. (1922). La pensée et la langue. Méthode, principes et plan d'une théorie nouvelle du langage appliquée au français. Paris : Masson et Compagnie.

BUSTARRET, C. (2011). « Couper, coller dans les manuscrits de travail du XVIII ${ }^{\mathrm{e}}$ au XX ${ }^{\mathrm{e}}$ siècle ». In : Jacob, C. (dir.), Lieux de savoir. Paris : Albin Michel, p. 353-375. 
CHARLE, C. \& VERGER, J. (2012). Histoire des universités : XII ${ }^{e}-X X I^{e}$ siècle. Paris : Presses universitaires de France.

CHEPIGA, V., EGUCHI, Y., FENOGLIO, I. \& LEFEBVRE, J. (2012). « Trois types discursifs pour une seule problématique théorique. Le couple conceptuel "sémiotique/sémantique" dans les manuscrits d'Émile Benveniste ». In : $3^{e}$ Congrès Mondial de Linguistique Française, vol. 1, p. 1057-1071.

CHEPIGA, V. \& SOFIA, E. (dirs) (2014). Archives et manuscrits de linguistes. Louvain-La-Neuve : Academia-L'Harmattan.

CHERVEL, A. (1988). « L'histoire des disciplines scolaires : réflexions sur un domaine de recherche ». Histoire de l'éducation 38, p. 59-119.

CHISS, J.-L., SAVATOVSKY, D., CANDEL, D. \& LÉON, J. (dirs) (2012). « La disciplinarisation des savoirs linguistiques. Histoire et épistémologie ». Les dossiers de HEL [supplément électronique à la revue Histoire Épistémologie Langage] 5.

DOQUET-LACOSTE, C. (dir.) (2009). «Écritures scientifiques. Carnets, notes, ébauches ». Langage et société 127 (1).

FENOGLIO, I. (coord.) (2012). « Le geste linguistique ». Genesis. Manuscrits, Recherche, Invention 35.

FOREL, C. (2008). La linguistique sociologique de Charles Bally :étude des inédits. Genève : Droz.

FRÝBA-REBER, A.-M. (2013). Philologie et linguistique romanes : institutionnalisation des disciplines dans les universités suisses (1872-1945). Louvain : Peeters.

JAСОВ, C. (dir.) (2011). Lieux de savoir. Paris : Albin Michel.

MEILLET, A. (1923). Les origines indo-européennes des mètres grecs. Paris : Presses universitaires de France.

MELI, C. (2014). « Bossuet : currente calamo, viva voce, entretien avec Gilles Philippe ». Genesis. Manuscrits, Recherche, Invention 39, p. 93-100.

MONDADA, L. (2005). Chercheurs en interaction: comment émergent les savoirs. Lausanne: Presses polytechniques et universitaires romandes.

NOGUÈs, B. (2008). «Élèves ou auditeurs ? Le public des facultés de lettres et de sciences au XIX ${ }^{\mathrm{e}}$ siècle (1808-1878) ». Histoire de l'éducation 120, p. 77-97.

PHILIPPE, G. (2014). «Écrire pour parler. Quelques problématiques premières ». Genesis. Manuscrits, Recherche, Invention 39, p. 11-28.

PUECH, C. \& CHISS, J.-L. (1998). « De l'émergence disciplinaire à la didactisation des savoirs linguistiques : le tournant des années 60 et ses suites ». Langue française 117 (1), p. 6-21.

VENDRYES, J. (dir.) (1953). Airne Fíngein. Dublin : Institute for Advanced Studies.

WAQUET, F. (2015). L'ordre matériel du savoir : comment les savants travaillent : XVI ${ }^{e}-X X I^{e}$ siècles. Paris : CNRS Éd.

\section{NOTES}

1. Cf. par exemple V. Chepiga et al. (2012) qui ont analysé dans les manuscrits d'É. Benveniste le traitement, à peu de temps d'intervalle, d'une même problématique (le couple sémiotique/ sémantique) dans trois genres discursifs distincts : la préparation d'une conférence scientifique, d'un article et d'un cours. 
2. Cf. par exemple, C. Bally (1930), J. Vendryes (1953), É. Benveniste (1969).

3. Ces manuscrits sont conservés dans différentes institutions. Les papiers Arsène Darmesteter appartiennent aux archives de l'Université de Paris et sont conservés à la Bibliothèque Interuniversitaire de la Sorbonne, sous la cote II Ms AUC 41. Le fonds Ferdinand Brunot se trouve à la bibliothèque de l'Institut de France. Il comporte dix cartons concernant sa formation et sa carrière d'enseignant sous les cotes Ms 7776 à Ms 7785. Les archives de Charles Bally sont à la Bibliothèque de Genève sous les cotes BGE Ms. fr. 5001-5153. Nous adressons nos remerciements à la Commission des bibliothèques et archives de l'Institut de France ainsi qu'à son président, Madame H. Carrère d'Encausse, secrétaire perpétuel de l'Académie française, qui nous ont permis d'exploiter le fonds Ferdinand Brunot. Nos remerciements vont également à la Bibliothèque Interuniversitaire de la Sorbonne pour nous avoir donné accès aux papiers Arsène Darmesteter ainsi qu'à la Bibliothèque de Genève et à la Société Académique de Genève pour l'autorisation qui nous a été faite de reproduire des manuscrits du fonds Charles Bally. Enfin, nous remercions chaleureusement $\mathrm{C}$. Forel pour sa générosité et son aide précieuse dans l'exploration des papiers de C. Bally.

4. G. Philippe (2014) note que l'une des caractéristiques des écrits préparant de l'oral - ce qu'il appelle des « avant-dire » - est l'alternance de segments rédigés et de segments notationnels non nécessairement liée à différentes phases de genèse. Cette alternance se rencontre effectivement dans plusieurs dossiers préparatoires à des cours universitaires.

5. C'est ce constat qui poussa C. Bally et A. Sechehaye, après l'examen des papiers de travail de F. de Saussure, à fonder leur édition du Cours de linguistique générale sur les cahiers d'étudiants.

6. Sur l'évolution du système universitaire français pour la période qui nous intéresse (1870-1930), on consultera C. Charle \& J. Verger (2012) ainsi que B. Noguès (2008). Sur la place de l'enseignement de la linguistique dans le système universitaire français à la fin du XIX ${ }^{\mathrm{e}}$ et au début du XX $X^{\mathrm{e}}$ siècle, on consultera les travaux de G. Bergounioux $(1984,1990,1998)$; pour un état des lieux institutionnel de l'enseignement de la linguistique en Suisse à la même période : voir A.M. Frýba-Reber (2013).

7. On pourrait même faire l'hypothèse que c'est une pratique ordinaire pour la préparation de tout genre oral récurrent. En tout cas, la pratique de réemploi matériel est attestée dans les manuscrits autographes des sermons de J. B. Bossuet, voir C. Meli (2014, p. 95).

8. Les notes préparatoires à ces cours sont conservées à la Bibliothèque de Genève sous les cotes Ms. fr. 5025-5051. C. Forel (2008) a étudié et publié une partie de ces manuscrits, ceux consacrés aux cours de « linguistique sociologique ». Son ouvrage constitue un vade-mecum précieux pour l'exploration des notes de cours de C. Bally. On trouvera aux pages 699 à 707 de cet ouvrage, le programme des cours donnés par C. Bally et l'index des manuscrits qui s'y rapportent.

9. Entre ces deux dates, C. Bally fait paraître sur le même sujet un important article dans la Revue internationale de sociologie: "La contrainte sociale dans le langage» (1927), repris dans la troisième édition du volume Le langage et la vie.

10. Ainsi, la meilleure connaisseuse de ces papiers, C. Forel (2008, p.107-108), a acté l'impossibilité de reconstituer l'ordre écrit, dans sa présentation des manuscrits des cours de linguistique sociologique: «Il aurait été heureux de pouvoir organiser la présentation de ces notes manuscrites en reconstituant ce que Bally projetait de professer dans chaque cours, mais il faut y renoncer, l'entreprise s'avérant compliquée et hasardeuse. À cause de ces difficultés, déplacements de matériel, remaniements impossibles à repérer, contenus indiqués pour un même cours dispersés dans plusieurs manuscrits, nous nous proposons d'exposer les différentes parties des manuscrits telles qu'elles se présentent à nous ".

11. L'ensemble comporte 45 feuillets. Plusieurs portent le même numéro.

12. L'utilisation de fiches et de fichiers dans la préparation à l'écriture et à l'oralisation se répand très largement parmi les intellectuels à partir de la fin du XIX ${ }^{\mathrm{e}}$ siècle. Sur ce point, voir $\mathrm{F}$. Waquet (2015, p. 74-89). 
13. Les annuaires de la $4^{\mathrm{e}}$ section de l'EPHE, celle des sciences historiques et philologiques, signalent le latin populaire en tant que thème d'étude dans les conférences d'A. Darmesteter presque chaque année de 1873-1874, soit sa seconde année d'enseignement dans cette institution, à sa dernière année, en 1881-1882. http://www.persee.fr/web/ouvrages/home/prescript/fond/ ephe.

14. En raison du manque de cohérence des numérotations successives d'A. Darmesteter, on fera référence ici à l'ordre dans lequel sont actuellement conservés les feuillets, qu'on numérotera de 1 à 20.

15. Nous soulignons.

16. Nous soulignons.

\section{RÉSUMÉS}

Chez certains enseignants-chercheurs de la fin du $\mathrm{XIX}^{\mathrm{e}}$ et du début du XX ${ }^{\mathrm{e}}$ siècle, préparer un cours universitaire implique de réutiliser des notes préexistantes. Après avoir exposé les problèmes méthodologiques que soulève l'identification de cette pratique dans les manuscrits conservés, on en propose une interprétation dans une perspective didactique. À partir d'exemples tirés des notes de C. Bally, F. Brunot et A. Darmesteter. On montre que des procédés tels que le déplacement, le découpage et le collage de feuillets préexistants témoignent non seulement de méthodes de travail propres à chaque enseignant, mais aussi d'une même volonté de concilier les exigences de l'enseignement et celles de la recherche. À travers des opérations de sélection et de restructuration, le processus d'écriture du cours révèle des tensions, à différentes échelles, entre l'aspect discursif et l'aspect matériel du texte.

For some academics involved in both teaching and research at the end of the $19^{\text {th }}$ and at the beginning of the $20^{\text {th }}$ century, preparing a university lecture entails reusing preexistent notes. After describing the methodological issues that arise when attempting to identify this practice in preserved manuscripts, we offer an interpretation from a didactical standpoint. Based on examples taken from notes by C. Bally, F. Brunot and A. Darmesteter, we show that procedures such as rearranging, cutting up and gluing preexistent leaves testify not only to each professor's own working methods, but also to a common intent to match the requirements of both teaching and research. Through operations of selection and restructuration, the process of writing a lecture reveals tensions, on various scales, between the text as discourse and the text as a material object.

\section{INDEX}

Mots-clés : écriture, enseignement, manuscrits, université, Charles Bally, Ferdinand Brunot, Arsène Darmesteter

Keywords : writing, teaching, manuscripts, university, Charles Bally, Ferdinand Brunot, Arsène Darmesteter 


\section{AUTEURS}

MURIEL JORGE

Université Sorbonne-Nouvelle-Paris 3, HTL, UMR 7597, F-75013, France

\section{PIERRE-YVES TESTENOIRE}

Université Paris-Sorbonne, ESPÉ de Paris, HTL, UMR 7597, F-75013, France 